\title{
Endovascular Management of Long-Segmental Petrocavernous Internal Carotid Artery (Carotid S) Occlusion
}

\author{
Soonchan Park, ${ }^{\mathrm{a}, \mathrm{b}}$ Eun Suk Park, ${ }^{\mathrm{c}}$ Jae Hyuk Kwak, ${ }^{\mathrm{a}}$ Dong-Geun Lee, ${ }^{\mathrm{a}}$ Dae Chul Suh, ${ }^{\mathrm{a}}$ Sun U. Kwon, ${ }^{\mathrm{d}}$ \\ Deok Hee Lee ${ }^{a}$ \\ ${ }^{a}$ Department of Radiology and Research Institute of Radiology, University of Ulsan College of Medicine, Asan Medical Center, Seoul, Korea \\ bepartment of Radiology, Kyung Hee University Hospital at Gangdong, School of Medicine, Kyung Hee University, Seoul, Korea \\ 'Department of Neurosurgery, Ulsan University Hospital, University of Ulsan College of Medicine, Ulsan, Korea \\ ${ }^{\mathrm{d}}$ Department of Neurology, University of Ulsan College of Medicine, Asan Medical Center, Seoul, Korea
}

Background and Purpose Long-segmental thrombotic occlusion of the distal internal carotid artery (ICA) sparing the cervical segment proximally and the supraclinoid segment distally, which could be termed 'Carotid S occlusion', has an unusual clinical presentation. However, endovascular management of this lesion is challenging. The purpose of our study is to report our endovascular treatment clinical experience of the disease.

Methods From March 2008 to June 2013, we could identify 14 patients (average age: 62.1, median age: 62, range: 50-79) with 'Carotid S occlusion', who underwent endovascular recanalization procedures. Patient's clinical presentations were collected and the imaging findings also analyzed. The technical success rate, 24-hour and follow-up imaging outcome, and the clinical outcome using the 90-day mRS (modified Rankin scale) score were evaluated.

Results Patients presented with gradually progressing $(n=8)$, fluctuating $(n=3)$, transient ischemic attack $(n=2)$ and stationary $(n=1)$ symptoms. DWI showed internal and external border-zone lesions in six patients, only internal ICA border-zone lesions in three patients, and only external border-zone lesions in two patients. Underlying distal ICA stenosis was noted in 12 patients. The technical success rate was $92.8 \%$ (13/14). Luminal patency was noted in all patients (100\%) after 24 hours and in nine of $10(90 \%)$ on long-term follow-up (median: 6.5, average: 15.1, range: 1-39 months). A 90-day, good functional outcome $(\mathrm{mRS} \leq 2)$ was noted in 13 of 14 patients $(92.8 \%)$.

Conclusions 'Carotid S occlusion' usually presented with border-zone infarction and endovascular management of the lesions was feasible. A relatively successful clinical outcome could be achieved after successful revascularization.

Keywords Stroke; Carotid artery, internal; Atherosclerosis; Angioplasty, balloon; Stents

\author{
Correspondence: Deok Hee Lee \\ Department of Radiology, Asan Medical \\ Center, 88 Olympic-ro 43-gil, Songpa-gu, \\ Seoul 05505, Korea \\ Tel: $+82-2-3010-5944$ \\ Fax: +82-2-476-0090 \\ E-mail:dhlee@amc.seoul.kr
}

Received: March 17, 2015

Revised: July 5, 2015

Accepted: July 6, 2015

The authors have no financial conflicts of interest.

\section{Introduction}

Acute occlusion of the internal carotid artery (ICA) may exhibit various clinical and radiographic features, according to the causes and anatomic sites of the occlusion. Unlike occlusions of the carotid $\mathrm{T}$ region or the proximal ICA, occlusion of the middle portion of the ICA, sparing the proximal cervical and supraclinoid segments, may show a different clinical presentation. For 
this disease entity, we have coined a new term, "carotid S occlusion," in view of the relative preservation of the collaterals to the ipsilateral anterior and middle cerebral arteries, which are supplied by collaterals of the retrograde ophthalmic artery and/or the circle of Willis. Endovascular management of this kind of segmental lesion is challenging, because of the long segment involvement, the frequent combination of underlying atherosclerosis with de novo thrombosis, and the high chance of distal clot migration during the procedure.

The purposes of this study were: 1 ) to define this unique clinical condition, i.e., "carotid S occlusion"; 2) to present its characteristic clinical and radiologic features; and 3) to report our clinical experience of the endovascular treatment of these lesions, together with procedural suggestions for successful recanalization.

\section{Methods}

\section{Definition of "carotid S occlusion"}

"S" denotes carotid siphon. Unlike distal ICA occlusion involving the $\mathrm{T}$ region, or occlusion of the proximal ICA involving the bulb or proximal cervical segment of the ICA, occlusion of the middle portion of the ICA, namely the carotid siphon or petrocavernous segment, shows peculiar clinical and radiologic features, primarily due to the relatively higher chance of recruiting

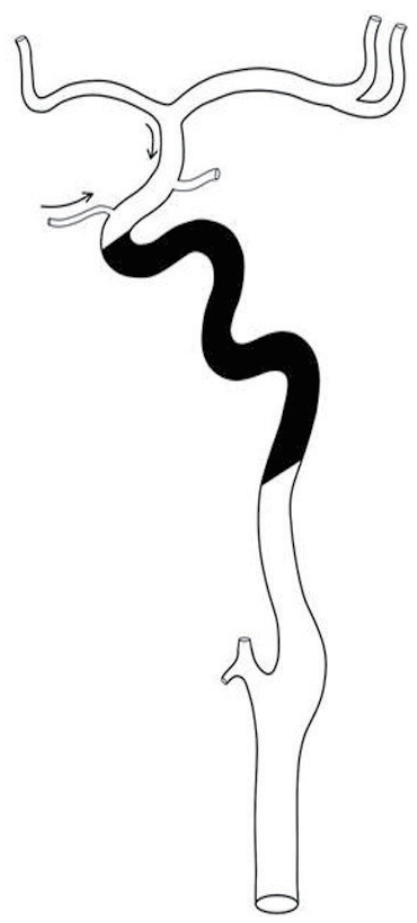

Figure 1. On angiography "carotid S occlusion" can be characterized by the following features. Long-segmental occlusion from the distal cervical segment to the anterior genu or horizontal portion of the cavernous ICA (marked in black) with collaterals via the ophthalmic artery and/or the circle of Willis via the anterior or posterior communicating arteries (curved arrows). collateral circulation from the retrograde flow of the ophthalmic artery and/or the circle of Willis. On catheter angiography, "carotid S occlusion" can be characterized by the following features (Figure 1):

1) Non-visualization of a long segment of the ICA from the distal cervical segment to the anterior genu or horizontal portion of the cavernous ICA;

2) The proximal ICA bulb and cervical segment look occluded but are patent, although there may be partial contrast filling due to the secondary collapse of the distal cervical segment;

3) The supraclinoid segment of the ICA is filled via the ophthalmic artery collateral segment. In some situations the collateral flow may fill the anterior genu or part of the horizontal portion of the cavernous ICA in retrograde fashion; and

4) Circle-of-Willis collaterals contribute to the intracranial flow, via the anterior communicating artery and/or the posterior communicating artery.

\section{Patients}

The inclusion criteria were (1) vascular imaging demonstrating "carotid S occlusion" on digital subtraction angiography and (2) acute or subacute ischemic stroke in the hemispheric territory of an occluded ICA. We drew subjects for this study from our neuro-interventional database from March 2008 to June 2013. Acute and subacute stroke were defined according to a symptom onset time of 6-48 hours and 48 hours to two weeks, respectively.

\section{Imaging}

Initial imaging was performed using multimodal magnetic resonance imaging (MRI) in most cases, according to the initial imaging protocol for acute ischemic stroke used in our medical institution. One patient underwent computed tomography (CT) imaging because the MR scanner was unavailable at that time. MRI was performed using a $1.5 \mathrm{~T}$ MR scanner (Avanto; Siemens Healthcare, Erlangen, Germany) with an 8-channel sensitivityencoding head coil. The sequences for our acute stroke, multimodal MR protocol included diffusion-weighted imaging (DWI) (acquisition matrix $=384 \times 384, \mathrm{TR}=3000 \mathrm{~ms}, \mathrm{TE}=86 \mathrm{~ms}$ ), conventional $\mathrm{T}^{*}$ gradient-echo imaging (GRE) (acquisition matrix $=256 \times 256$, TR $=690 \mathrm{~ms}$, TE $=16 \mathrm{~ms}$ ), fluid-attenuated inversion recovery (FLAIR) (acquisition matrix $=256 \times 224$, $\mathrm{TR}=9,000 \mathrm{~ms}, \mathrm{TE}=100 \mathrm{~ms}$ ), dynamic susceptibility MR perfusion (acquisition matrix $=128 \times 128, \mathrm{TR}=1,407 \mathrm{~ms}, \mathrm{TE}=40$ $\mathrm{ms}$ ), time-of-flight $\mathrm{MR}$ angiography (acquisition matrix = $640 \times 640, \mathrm{TR}=24 \mathrm{~ms}, \mathrm{TE}=7 \mathrm{~ms}$ ), and contrast-enhanced magnetic resonance angiography (MRA) (acquisition matrix $=$ $640 \times 640, \mathrm{TR}=3.7 \mathrm{~ms}, \mathrm{TE}=1.3 \mathrm{~ms})$. The total acquisition time was less than 20 minutes, including the patient handling time. 


\section{Indications of revascularization}

The patients with acute stroke who presented within six hours of onset of their symptoms were managed according to our institution's acute stroke management protocol. ${ }^{1}$ Endovascular therapy was offered to patients who met the following criteria: 1) acute stroke with no significant response to intravenous tissue plasminogen activator (IV tPA) infusion; 2) acute stroke with indications not for IV tPA but for intra-arterial thrombolysis; and 3) subacute stroke with limited DWI high-signal lesion volume along the ICA border-zone and with significant symptoms and/or diffusion-perfusion mismatch. ${ }^{2}$ In patients with a subacute presentation, we applied very conservative indications in terms of the DWI lesion volume, in order to avoid the possibility of reperfusion injury in a larger infarction. We did not include any patient with hemorrhage demonstrated by gradient echo images on MRI or non-enhanced CT.

\section{Conventional angiography and revascularization procedures}

After obtaining informed patient consent for performing endovascular revascularization procedures, we administered loading doses of dual antiplatelets (300-500 mg aspirin, $300 \mathrm{mg}$ clopidogrel) in subacute patients and continued with the procedure without any oral antiplatelet medication in acute cases. In those patients, a loading dose of tirofiban (Aggrastat; Merck \& Co., Inc., Whitehouse Station, NJ, USA) was given intravenously immediately before a stent was placed.

After the occlusion sites, pattern, and length had been confirmed, revascularization procedures were begun with the patient under local anesthesia. When a patient was uncooperative, conscious sedation was achieved using propofol (Diprivan; Hospira, Inc., Lake Forest, Il, USA). Systemic heparinization with a target activated clotting time of 200-250s was achieved in subacute cases. In an acute setting, a reduced dose of heparin (2,000-3,000 U bolus, $500 \mathrm{U} / \mathrm{h}$ thereafter) was administered at the beginning of the procedure.

In the later phase of our clinical experience, we used a 9-Fr, balloon-tipped guiding catheter (Optimo; Tokai Medical Products, Japan), the tip of which was placed in the bulb portion or above so that we could occlude the cervical segment of the ICA by inflating the occlusion balloon. Suction thrombectomy through the catheter was then attempted by manually applying negative pressure with the use of a 50-mL syringe. However, during the earlier phase of our clinical experience, before the balloon-tipped guiding catheter was available, ICA flow arrest was achieved by external manual compression of the carotid artery $(\mathrm{n}=2)$ while a 7-8-F guiding catheter was placed as distally as possible up into the sub-petrosal portion of the ICA, so as to avoid inadvertent distal migration of the fragmented clot debris. After a stentriever device (Solitaire AB or FR; Covidien, Irvine, CA, USA) became available, clot retrieval was attempted when manual aspiration thrombectomy was not effective.

After attempting several suction aspirations and/or passage of stentrievers, we checked the recanalization status of the ICA by observing not just the retrieved clots, but also the spontaneous backflow of fresh carotid blood through the proximal tip of the unlocked guiding catheter hub. If significant regurgitation of blood was noted and non-resistant gentle aspiration of the catheter was possible without further visualization of the aspirated clot, the occlusion balloon was then deflated so that we could check the recanalization status.

If there was no underlying stenosis or filling defect and improvement of the antegrade flow was observed, we completed the procedure. If there was no backflow of blood or any interruption of blood aspiration on gentle suction by the guiding catheter, a large-bore microcatheter (Prowler select plus; Codman, Excelsior XT27, or Renegade; Stryker Neurovascular) was then advanced up to the petrous portion in order to perform a careful retrograde control angiogram of the ICA while the guiding catheter hub was wide open. If there was neither any filling defect nor stenosis, the microcatheter tip was advanced more distally in order to check the distal ICA patency, and the step was repeated until we could find the cause of the persistent occlusion.

If there was any flow-limiting stenosis or filling defect seen on the retrograde control angiography, we then placed a self-expanding stent (Wingspan System; Stryker Neurovascular, Neuroform; Stryker Neurovascular, Enterprise; Codman) after predilatation of the lesion using a balloon catheter (Maverick; Boston Scientific Corp., or Gateway; Stryker Neurovascular). The stent was chosen according to the nature of the lesion. Sometimes we needed to place a stent to stabilize filling defects-i.e., clots stuck to the wall but not responding to the aspiration and clot retrieval —in the stenotic segment, even though the stenosis was not flow limiting.

\section{Post-procedural management and imaging}

After completing the procedure, we transferred the patient to the neuro-intensive care unit without reversing the heparin. The patient's blood pressure was closely monitored and adjusted in order to prevent hyperperfusion syndrome. Non-contrast head $\mathrm{CT}$ and $\mathrm{CT}$ angiography (CTA) were performed 24 hours after the procedure. Any patient who showed re-occlusion or newly developed symptoms was advised to undergo the reperfusion procedures again, taking into account the infarct volume. Early follow-up multi-modal MRI was performed approximately 3-5 days after the procedure for the evaluation of infarction evolution. 
Table 1. Initial clinical characteristics of the patients

\begin{tabular}{|c|c|c|c|c|c|c|c|c|c|c|c|c|c|}
\hline Pt No. & Sex/age & HTN & DM & $\mathrm{HL}$ & SM & C & CES & Sx & Stage & TOAST & Onset (hour) & $\mathrm{NIHSS}_{0}$ & Initial mRS \\
\hline 1 & $\mathrm{M} / 51$ & $(-)$ & $(-)$ & $(-)$ & $(+)$ & $(-)$ & $(-)$ & GP & acute & $\angle A D$ & 12 & 6 & 4 \\
\hline 2 & M/68 & $(+)$ & $(+)$ & $(+)$ & $(+)$ & $(-)$ & $(-)$ & TIA & subacute & $L A D$ & 120 & 8 & 4 \\
\hline 3 & $M / 53$ & $(+)$ & $(-)$ & $(+)$ & $(-)$ & $(-)$ & $(-)$ & $\mathrm{TIA}$ & acute & LAD & 28 & 4 & 2 \\
\hline 4 & $M / 69$ & $(-)$ & $(-)$ & $(-)$ & $(+)$ & $(-)$ & $(-)$ & GP & acute & $L A D$ & 48 & 3 & 2 \\
\hline 5 & $F / 61$ & $(+)$ & $(-)$ & $(-)$ & $(-)$ & $(-)$ & $(-)$ & GP & acute & LAD & 32 & 8 & 4 \\
\hline 6 & $M / 56$ & $(+)$ & $(+)$ & $(-)$ & $(+)$ & $(-)$ & $(-)$ & $S$ & subacute & LAD & 240 & 2 & 1 \\
\hline 7 & $F / 79$ & $(+)$ & $(+)$ & $(-)$ & $(-)$ & $(-)$ & SSS & GP & acute & $\mathrm{CE}$ & 6 & 17 & 4 \\
\hline 8 & $\mathrm{M} / 52$ & $(-)$ & $(+)$ & $(+)$ & $(+)$ & $(-)$ & $(-)$ & $\mathrm{F}$ & subacute & $L A D$ & 240 & 2 & 2 \\
\hline 9 & $\mathrm{M} / 50$ & $(-)$ & $(+)$ & $(+)$ & $(-)$ & $(-)$ & $(-)$ & GP & subacute & $L A D$ & 107 & 16 & 4 \\
\hline 10 & $F / 72$ & $(-)$ & $(+)$ & $(+)$ & $(-)$ & $(-)$ & $(-)$ & GP & acute & CE & 9 & 15 & 4 \\
\hline 11 & $\mathrm{M} / 58$ & $(-)$ & $(-)$ & $(-)$ & $(-)$ & $(-)$ & $(-)$ & $\mathrm{F}$ & subacute & LAD & 110 & 12 & 3 \\
\hline 12 & $\mathrm{M} / 62$ & $(-)$ & $(-)$ & $(-)$ & $(+)$ & $(+)$ & $(-)$ & $\mathrm{GP}$ & acute & $\angle A D$ & 8 & 5 & 3 \\
\hline 13 & M/65 & $(+)$ & $(+)$ & $(-)$ & $(+)$ & $(-)$ & $(-)$ & $\mathrm{F}$ & subacute & LAD & 140 & 3 & 1 \\
\hline 14 & $F / 74$ & $(+)$ & $(-)$ & $(-)$ & $(-)$ & $(-)$ & $(-)$ & GP & acute & $L A D$ & 44 & 7 & 4 \\
\hline
\end{tabular}

Pt indicates patient; No., number; HTN, hypertension; DM, diabetes mellitus; HL, hyperlipidemia; SM, smoking; C, coronary heart disease; CES, cardiac embolic source; Sx, symptom; TOAST, Trial of Org 10172 in the Acute Stroke Treatment classification; NIHSS ${ }_{0}$, the National Institutes of Health Stroke Scale on admission; mRS modified RankinScore; SSS, sick sinus syndrome on pacemaker; GP, gradually progressing; TIA, multiple transient ischemic attack; S, stationary; F, fluctuating; LAD, large artery disease; CE, cardioembolism.

\section{Data collection and analysis}

The patients' baseline characteristics, including demographic data, are summarized in Table 1 . The stroke severity was checked using the National Institutes of Health Stroke Scale on admission and the modified Rankin scale (mRS) score for clinical followup. The stroke presentation (acute or subacute) and pattern (improving, stationary, gradually worsening or fluctuating), including the ischemic duration, were recorded. To evaluate the etiology of the occlusion, patients with concomitant heart disease, vascular risk factors, and a history of stroke or coronary heart disease were identified and classified according to Trial of Org 10172 in the Acute Stroke Treatment (TOAST) classification.

We analyzed the initial CT, MRI, and digital subtraction angiography findings, focusing on the stroke pattern on cross-sectional images and the angiographic pattern on both MRA and digital subtraction angiography. We analyzed the DWI high-signal lesion pattern: e.g., external border-zone infarction, internal border-zone infarction, cortical wedge-shaped infarction, and basal ganglia involvement. For the angiographic findings, we analyzed the status of the proximal ICA patency by observing whether the shape of the bulb was normal or stenotic. Any collaterals present for ipsilateral intracranial ICA territorial flow were identified as retrograde ophthalmic artery collateral or circle-of-Willis collateral.

Technical success was defined as achievement of a TIMI grade $\geq 2$ in the occluded ICA. Procedural findings were analyzed in relation to the presence of combined thrombosis, the presence and anatomic location of underlying stenosis, response to aspiration/mechanical thrombectomy, application of balloon angioplasty and the response, application of a stent and detailed speci- fication of the stent used, application of post-stent angioplasty, and the presence and degree of residual stenosis seen on final control angiography. Residual stenosis was defined as the difference between the normal reference segment diameter and the minimum lumen diameter of the culprit lesion after intervention, divided by the reference segment diameter. Occurrence of any procedure-related complication was identified and the causes of procedural failure were identified.

The procedural outcome was monitored with CTA, which was performed 24 hours after the procedure in most patients. In two patients, MRA was performed with or without transcranial Doppler monitoring. Luminal patency was evaluated by direct demonstration of the ICA lumen with CTA. The MRA patency criteria were strong distal arterial flow signals similar to those on the contralateral side. Transcranial Doppler was used as a complementary tool. The 24-hour patency rate was calculated. We performed re-intervention in some patients, and the reasons for re-intervention were addressed.

The 90-day functional outcome was analyzed in terms of the $\mathrm{mRS}$, with a good outcome being defined as a score $\leq 2$.

\section{Results}

\section{Clinical and imaging characteristics of the patients}

We were able to identify 14 patients with "carotid S occlusion" who underwent endovascular recanalization procedures. There were 10 male and four female patients with a median age of 62 (IQR, 52.75-69.75). The patients' clinical and imaging characteristics are summarized in Tables 1 and 2. The median National Institutes of Health Stroke Scale on admission was 7 (range, 
Table 2. Imaging characteristics and follow-up results

\begin{tabular}{|c|c|c|c|c|c|c|c|c|c|}
\hline Pt No. & Angioplasty & Stenting & DWI & OphA & COW & Stenosis site & $\mathrm{mRS}$ at discharge & $\mathrm{mRS}$ at $3 \mathrm{M}$ & Clinical F/U period (M) \\
\hline 1 & $(-)$ & $(+)$ & $E$ & $(+)$ & $(+)$ & V & 0 & 0 & 66 \\
\hline 2 & $(+)$ & $(+)$ & $E, I$ & $(+)$ & $(+)$ & $\mathrm{L}$ & 2 & 1 & 2 \\
\hline 3 & $(+)$ & $(+)$ & $E, I, B$ & $(+)$ & $(+)$ & $\mathrm{L}$ & 2 & 0 & 41 \\
\hline 4 & $(-)$ & $(+)$ & E & $(+)$ & $(+)$ & $A G$ & 1 & 1 & 41 \\
\hline 5 & $(+)$ & $(+)$ & $E, I$ & $(+)$ & $(-)$ & $A G$ & 2 & 1 & 40 \\
\hline 6 & $(+)$ & $(+)$ & $E, I, C$ & $(+)$ & $(+)$ & $P G$ & 1 & 1 & 33 \\
\hline 7 & $(-)$ & $(-)$ & NA & $(+)$ & $(+)$ & $(-)$ & 3 & 4 & 7 \\
\hline 8 & $(+)$ & $(+)$ & $C$ & $(+)$ & $(+)$ & $\mathrm{L}$ & 2 & 0 & 11 \\
\hline 9 & $(+)$ & $(+)$ & I & $(+)$ & $(+)$ & $\mathrm{H}$ & 3 & 2 & 25 \\
\hline 10 & $(-)$ & $(-)$ & I & $(+)$ & $(+)$ & $(-)$ & 1 & 0 & 24 \\
\hline 11 & NA & NA & $\mathrm{T}$ & $(+)$ & $(+)$ & NA & 4 & 2 & 22 \\
\hline 12 & $(+)$ & $(-)$ & $E, I, C$ & $(+)$ & $(+)$ & $\mathrm{H}$ & 1 & 0 & 20 \\
\hline 13 & $(+)$ & $(+)$ & $E, I, C$ & $(+)$ & $(+)$ & $\mathrm{H}$ & 0 & 0 & 8 \\
\hline 14 & $(+)$ & $(-)$ & 1 & $(+)$ & $(-)$ & $\mathrm{H}$ & 2 & 2 & 7 \\
\hline
\end{tabular}

M indicates months; DWI, diffusion-weighted image; OphA, ophthalmic artery; COW, circle of Willis; mRS, modified Rankin-Score; F/U, follow-up; E, external border zone; I, internal border zone; C, cortical; B, basal ganglia; T, large territorial; H, horizontal cavernous; L, lacerum; AG, anterior genu; PG, posterior genu; V, vertical cavernous; NA; not available.

2-17). Mild proximal ICA stenosis was observed in two patients (No. 10 and 14). All but two patients had ophthalmic and circleof-Willis collaterals; these two patients had only ophthalmic collaterals. A tandem thromboembolic lesion beyond the ICA bifurcation was noted in two patients.

\section{Procedural findings}

The procedural characteristics are summarized in Table 2. Endovascular recanalization was successful in 13 of the 14 patients (92.8\%). IV tPA was not used in our patients because none met the indications; tirofiban was used in half of the patients.

\section{Causative underlying stenosis}

We were able to identify the underlying cause and anatomic site of a steno-occlusive lesion during or after the completion of the procedure. We observed underlying stenotic lesions in 12 of the 14 patients in whom recanalization procedures were attempted (85.7\%); the lesion sites are shown in Table 1.

\section{Thrombectomy results}

Among the 14 patients, aspiration thrombectomy was attempted in 12 patients. We did not perform aspiration thrombectomy in two patients as there was no thrombosis in the occluded segment $(n=2)$. Aspiration thrombectomy was effective in 33.3\% (4/12).

After it became available in our medical institution, Solitaire thrombectomy was performed in six patients as an adjunctive procedure, leading to recanalization in $83.3 \%(5 / 6)$. Although a hemodynamic cause plays a role in most stroke mechanisms, a large clot burden in the long-segmental occluded ICA can cause more serious infarctions. Therefore, if adjunctive thrombecto- my failed, we performed intentional internal trapping (patient No. 11). ${ }^{3}$

\section{Angioplasty and stenting}

Angioplasty was performed in nine patients and showed an improved luminal diameter in 88.9\% (8/9) and immediate reocclusion in $11.1 \%(1 / 9)$ of the patients. No stenting was needed after angioplasty in two patients. Stenting without angioplasty was done in two patients. Post-stenting angioplasty was not performed in any of the patients. One self-expandable stent was deployed in the stenotic distal ICA in each of six patients. Two stents were placed in each of three patients. We used the following stent combinations: Neuroform stent $(\mathrm{n}=1)$; double Neuroform stents $(\mathrm{n}=2)$; Enterprise stent $(\mathrm{n}=2)$; Neuroform and Enterprise stents $(\mathrm{n}=2)$; Wingspan stent $(\mathrm{n}=2)$; and Solitaire $A B$ stent $(n=1)$.

There was no stenosis in the distal ICA in patients No. 7 and 10. Concomitant stenting in the carotid bulb was performed in patients No. 13 and 14, as a large clot burden in this area created sluggish flow, despite recanalization of the distal ICA. There was residual stenosis $\geq 50 \%$ in $21.4 \%(3 / 11)$ in the distal ICA in the technically recanalized group.

\section{Procedure-related complications}

Distal clot migration was seen in patient No. 1 ( $\mathrm{n}=1,1$ out of 2) and proximal clot migration was visible in patients No. 3 and $10(\mathrm{n}=2)$. It is notable that in the distal migration case the procedure was performed without proximal flow control, whereas proximal flow control was used in all proximal migration cases. In patient No. 10, proximal migration was retrieved using the Solitaire system. In patient No. 14, a carotid cavernous fistula de- 

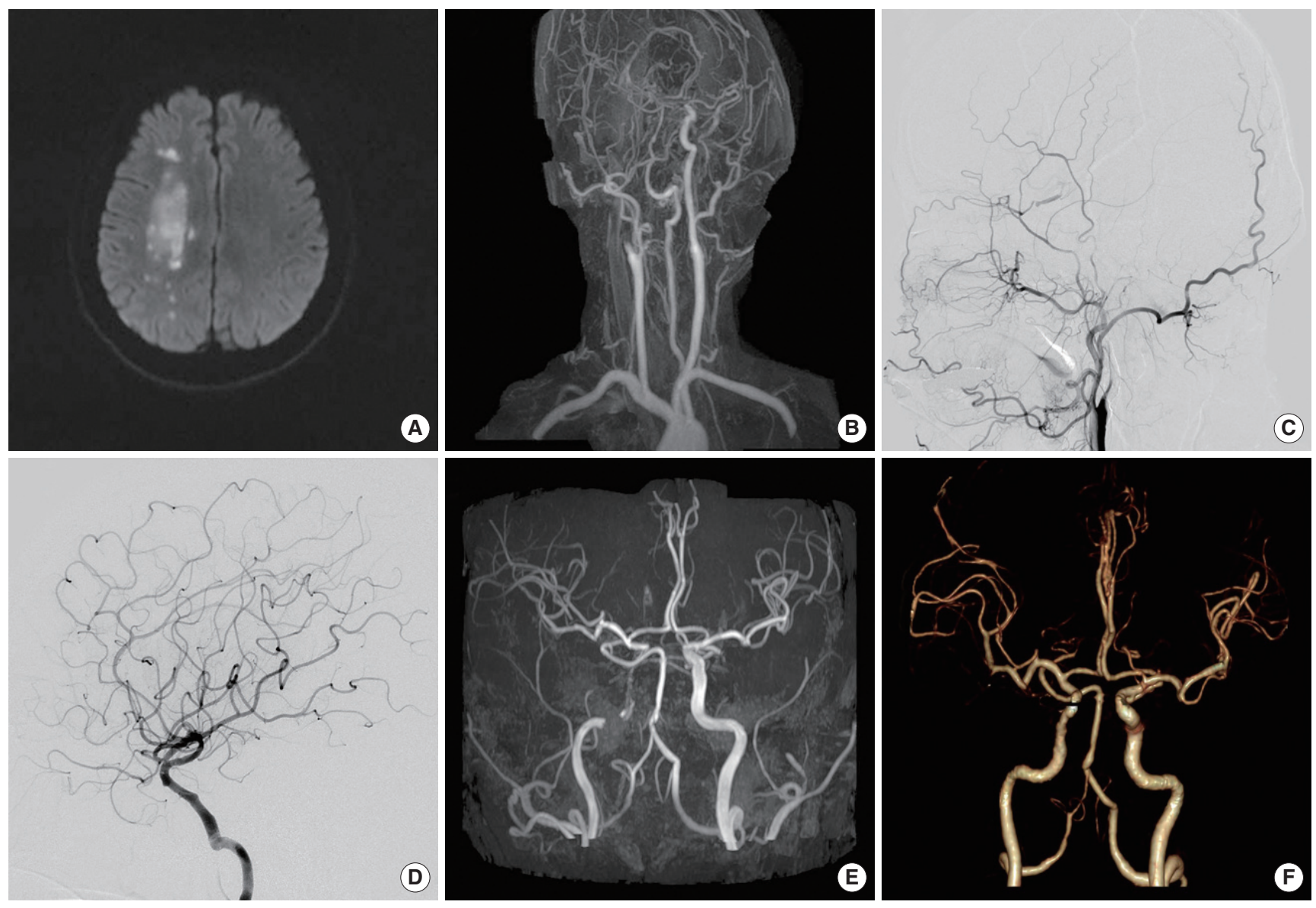

Figure 2. A 50-year-old male presented with gradually increasing left side weakness and aphasia. (A) Initial diffusion-weighted imaging shows an internal borderzone infarct lesion. (B) Contrast-enhanced magnetic resonance angiography shows occlusion of the right ICA from the cervical ICA just above the bulb to the cavernous segment. (C) Digital subtraction angiography shows occlusion of the right ICA with faint retrograde filling of the supraclinoid ICA. (D) Successful recanalization after suction thrombectomy and stenting shows recanalization of the ICA. (E) MRA performed four days later shows patent ICA flow. (F) Follow-up CTA two years later shows good patency of the stented segment.

veloped after pre-stenting angioplasty, but it spontaneously and immediately closed after balloon angioplasty in the segment.

\section{Short-term postprocedural imaging and additional procedures}

Twenty-four-hour imaging was performed in all recanalized patients $(\mathrm{n}=13)$ using CTA $(\mathrm{n}=11)$, computed tomography perfusion and DWI $(n=1)$, and MRI $(n=1)$. All patients showed patency of the recanalized ICA (Figure 2).

Patients No. 3 and 4 underwent repeated treatment for residual stenosis ( $20 \%$ and $60 \%$, respectively) noted on imaging. In patient No. 3, conventional angiography was performed because DWI showed new lesions and computed tomography perfusion showed a perfusion decrease one day later. We confirmed reocclusion and balloon angioplasty was performed. In patient No. 4, retreatment with balloon angioplasty was performed four days later, because of recurrent transient ischemic attacks and mean transit time delay seen on magnetic resonance perfusion, even though patency was shown on imaging. There was no intracranial hemorrhage related to the recanalization procedures. Two re-intervention sessions were required for patient No. 14. The first re-intervention was performed for recurrent carotid cavernous fistula five months later using covered stenting. An additional procedure was required as reocclusion occurred one month after treating the carotid cavernous fistula. This patient underwent recanalized thrombectomy using Penumbra, Solitaire and percutaneous transluminal angioplasty was performed for the stented segment. All re-interventions were completed without any serious adverse event.

\section{Follow-up results}

Imaging follow-up was performed in 10 patients (average: 15.1, median: 7, range: 1-39 months) (Table 1), nine of whom showed long-term patency. One patient, No. 2, showed reocclusion due to combined invasive aspergillosis of the left side of the cavernous sinus. 
There were no patient deaths. All patients were followed up clinically (average: 24.7 , median: 23 , range: $2-66$ months). A score of $m R S \leq 2$ at discharge was seen in $78.5 \%$ (11 out of 14 patients) and 90-day $\mathrm{mRS} \leq 2$ was seen in $92.8 \%$ (13 out of 14 patients). The patient treated with internal trapping (patient No. 11) also had an mRS of 2.

\section{Discussion}

Acute stroke caused by acute occlusion of the ICA is associated with poor outcomes. ${ }^{4}$ The MR CLEAN trial showed the clinical functional benefit of endovascular treatment in patients with anterior circulation acute stroke. ${ }^{5}$ Moreover, the outcome of ICA occlusion depends on the site of the occlusion during the endovascular treatment, because a robust collateral circulation is associated with better clinical outcomes. ${ }^{6}$ The rate of cervical ICA recanalization ranges from $84 \%$ to $100 \%$ with different recanalization strategies. ${ }^{7}$ However, pooled data from patients with acute cervical ICA occlusion and who underwent endovascular treatment showed a poor outcome in which nearly half of those with arterial recanalization remain functionally dependent. ${ }^{8}$ Carotid terminus occlusion has the worst functional outcome. ${ }^{9}$ Mechanical thrombectomy in carotid $\mathrm{T}$ occlusion showed a recanalization rate up to $86 \%{ }^{10}$ and a good clinical outcome in $39 \%-45 \%$ of the recanalized patients. ${ }^{411}$

Only a few reports have described recanalization of the petrocavernous ICA. ${ }^{12-14}$ The patient population was small ( 5 out of 31 ) and showed a low recanalization rate of $43 \% .{ }^{14}$ Therefore, this disease entity has not gained any special attention and there is no published study that assessed the imaging pattern, symptom presentation, treatment technique, and results for this disease entity. "Carotid S occlusion" is different from carotid terminus or proximal ICA occlusion in that symptoms progress gradually or fluctuate in most cases. For this reason, we had 6 subacute cases out of 14 . Furthermore, if it is recanalized, "Carotid S occlusion" shows a better clinical outcome than carotid terminus or proximal ICA occlusion. The radiological features are also different from those in occlusion of other ICA sites. The proximal ICA can be either visualized or not, depending on the thrombus burden. Ophthalmic collaterals fill the supraclinoid ICA but not the petro-cavernous ICA. Therefore, it is important to recognize this disease entity, as the appropriate treatment procedure is technically feasible and has relatively successful results, but only if the condition can be identified and treated.

We believe that the stroke mechanism of "carotid S occlusion" is mainly hemodynamic, as can be inferred from the fact that the infarct pattern is mostly border-zone and the predominant patient symptoms are gradual progression $(\mathrm{n}=8)$ and fluctuation $(\mathrm{n}=3)$. In patients with symptomatic ICA occlusion, the recruitment of ophthalmic collaterals is associated with hemodynamic impairment. However, this association is confounded by the inter-patient variability of the decrease in metabolic demand due to ischemic lesions. ${ }^{15}$ The rationale for the revascularization of an occluded ICA beyond the acute stage may be that the risk of ipsilateral stroke increases to $10 \%-20 \%$ per year when we consider the population with severe impairment of cerebrovascular hemodynamics. ${ }^{16,17}$

We had two patients with recurrent stroke. One (patient No. 4) had a transient ischemic attack one week later, which disappeared after balloon angioplasty. The other patient (No. 7) had contralateral side embolic infarction. This patient had the worst outcome, with an $\mathrm{mRS}$ of 4 at 3 months. The initial problem in recanalizing an occluded ICA is how we identify the occlusion point. We cannot be completely sure of the occlusion point without using digital subtraction angiography. MRA can only show long-segmental occlusion, and ophthalmic collaterals might not be sufficiently demonstrated on MRA. It is of great significance how we check the lumen while preventing distal clot migration. A microcatheter gradually advanced upwards can be used to check the occlusion level and the luminal patency while the hub of the guiding catheter is open to allow backward flow of the contrast medium, thus avoiding distal dislodgement of the clot.

The major risk of endovascular treatment is distal migration of thrombotic debris that could cause aggravation of a patient's symptoms. However, various protective devices have been developed to reduce embolic complications. ${ }^{18,19}$ Among these, we chose the proximal balloon because of its convenient use, highly successful performance, and the fact that the lesions we encountered deterred us from using distal protective devices. Proximal flow control implements the clot aspiration procedure that prevents distal clot migration to the intracranial vessels. ${ }^{20}$

It is important that, until complete revascularization has been confirmed, a proximal balloon should always be kept inflated in order to prevent clot migration. When we were performing this step without a balloon-tipped guiding catheter, we applied manual neck compression at the time we retrieved a clot. This technique may not be feasible in the case of a tortuous carotid artery or refractory clots, despite suction thrombectomy and a stentriever. In the earlier phase of our clinical experience, we had a case of inadvertent distal embolization of the clots, which was critical for the outcome of the procedure as balloon-tipped guiding catheters were not available. We did not observe any procedure-related hemorrhage or hyperperfusion. This may have been because of minor flow from the collateral before recanalization.

The main limitation of our study is that the number of enrolled patients was small; thus, further study is needed to vali- 
date our conclusions.

\section{Conclusions}

Long-segmental thrombotic occlusion of distal ICA sparing the cervical segment proximally and the supraclinoid segment distally, which we term "carotid S occlusion", usually presents with border-zone infarction with gradually progressing or fluctuating symptoms due to relatively preserved collaterals. Endovascular management of the lesions is feasible with the use of a balloon-tipped guiding catheter and self-expanding stents in patients with acute and subacute symptoms.

\section{References}

1. Cho AH, Sohn SI, Han MK, Lee DH, Kim JS, Choi CG, et al. Safety and efficacy of MRI-based thrombolysis in unclear-onset stroke. A preliminary report. Cerebrovasc Dis 2008;25:572-579.

2. Kang DW, Sohn SI, Hong KS, Yu KH, Hwang YH, Han MK, et al. Reperfusion therapy in unclear-onset stroke based on MRI evaluation (RESTORE): a prospective multicenter study. Stroke 2012;43:3278-3283.

3. Park JW, Lee DH, Choi CG, Kim SJ, Suh DC. Various endovascular approaches to the management of free floating carotid thrombi: a technical report. J Neurointerv Surg 2012;4:336-338.

4. Flint AC, Duckwiler GR, Budzik RF, Liebeskind DS, Smith WS. Mechanical thrombectomy of intracranial internal carotid occlusion: pooled results of the MERCI and Multi MERCI Part I trials. Stroke 2007;38:1274-1280.

5. Berkhemer OA, Fransen PS, Beumer D, van den Berg LA, Lingsma HF, Yoo AJ, et al. A randomized trial of intraarterial treatment for acute ischemic stroke. N Engl J Med 2015;372:11-20.

6. Liebeskind DS, Tomsick TA, Foster LD, Yeatts SD, Carrozzella J, Demchuk AM, et al. Collaterals at angiography and outcomes in the Interventional Management of Stroke (IMS) III trial. Stroke 2014;45:759-764.

7. Rahme R, Abruzzo TA, Ringer AJ. Acute ischemic stroke in the setting of cervical carotid occlusion: a proposed management strategy. World Neurosurg 2011;76:S60-65.

8. Seet RC, Wijdicks EF, Rabinstein AA. Stroke from acute cervical internal carotid artery occlusion: treatment results and predictors of outcome. Arch Neurol 2012:169:1615-1620.

9. Fischer U, Mono ML, Schroth G, Jung S, Mordasini P, El-Koussy M, et al. Endovascular therapy in 201 patients with acute symptomatic occlusion of the internal carotid artery. Eur J Neurol 2013;20:1017-1024, e87.
10. Lin R, Vora N, Zaidi S, Aleu A, Jankowitz B, Thomas A, et al. Mechanical approaches combined with intra-arterial pharmacological therapy are associated with higher recanalization rates than either intervention alone in revascularization of acute carotid terminus occlusion. Stroke 2009;40:2092-2097.

11. Hwang YH, Kang DH, Kim YW, Kim YS, Park SP, Suh CK. Outcome of forced-suction thrombectomy in acute intracranial internal carotid occlusion. J Neurointerv Surg 2013;5(Suppl 1): i81-84.

12. Terada T, Okada H, Nanto M, Shintani A, Yoshimura R, Kakishita K, et al. Endovascular recanalization of the completely occluded internal carotid artery using a flow reversal system at the subacute to chronic stage. J Neurosurg 2010;112:563-571.

13. Kao HL, Lin MS, Wang CS, Lin YH, Lin LC, Chao CL, et al. Feasibility of endovascular recanalization for symptomatic cervical internal carotid artery occlusion. J Am Coll Cardiol 2007;49: 765-771.

14. Siddiq F, Chaudhry SA, Das P, Khatri R, Rodriguez G, Qureshi AI. Occurrence and prognostic significance of cervical pseudodissection phenomenon associated with acute intracranial internal carotid artery occlusion. J Neuroimaging 2013;23: 384390.

15. Yamauchi H, Kudoh T, Sugimoto K, Takahashi M, Kishibe Y, Okazawa H. Pattern of collaterals, type of infarcts, and haemodynamic impairment in carotid artery occlusion. J Neurol Neurosurg Psychiatry 2004;75:1697-1701.

16. Grubb RL Jr, Derdeyn CP, Fritsch SM, Carpenter DA, Yundt $\mathrm{KD}$, Videen TO, et al. Importance of hemodynamic factors in the prognosis of symptomatic carotid occlusion. JAMA 1998; 280:1055-1060.

17. Kuroda S, Houkin K, Kamiyama H, Mitsumori K, Iwasaki Y, Abe H. Long-term prognosis of medically treated patients with internal carotid or middle cerebral artery occlusion: can acetazolamide test predict it? Stroke 2001;32:2110-2116.

18. Kastrup A, Gröschel K, Krapf H, Brehm BR, Dichgans J, Schulz JB. Early outcome of carotid angioplasty and stenting with and without cerebral protection devices: a systematic review of the literature. Stroke 2003;34:813-819.

19. Terada T, Tsuura M, Matsumoto H, Masuo O, Yamaga H, Tsumoto $\mathrm{T}$, et al. Results of endovascular treatment of internal carotid artery stenoses with a newly developed balloon protection catheter. Neurosurgery 2003;53:617-623.

20. Chueh JY, Wakhloo AK, Gounis MJ. Effectiveness of mechanical endovascular thrombectomy in a model system of cerebrovascular occlusion. AJNR Am J Neuroradiol 2012;33:1998-2003. 